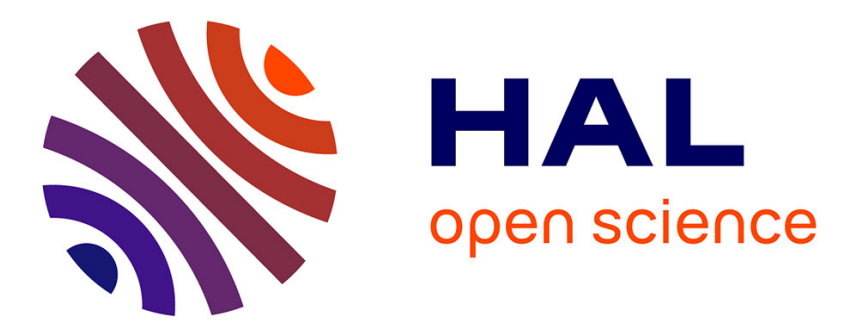

\title{
Evapotranspiration en zone semi-aride de deux couverts végétaux (gazon, blé) obtenue par plusieurs méthodes. I. - Evaluation de l'ETP (conditions hydriques non limitantes)
}

Charles Riou, Roger Chartier

\section{To cite this version:}

Charles Riou, Roger Chartier. Evapotranspiration en zone semi-aride de deux couverts végétaux (gazon, blé) obtenue par plusieurs méthodes. I. - Evaluation de l'ETP (conditions hydriques non limitantes). Agronomie, 1985, 5 (3), pp.261-266. hal-00884757

\section{HAL Id: hal-00884757 \\ https://hal.science/hal-00884757}

Submitted on 1 Jan 1985

HAL is a multi-disciplinary open access archive for the deposit and dissemination of scientific research documents, whether they are published or not. The documents may come from teaching and research institutions in France or abroad, or from public or private research centers.
L'archive ouverte pluridisciplinaire HAL, est destinée au dépôt et à la diffusion de documents scientifiques de niveau recherche, publiés ou non, émanant des établissements d'enseignement et de recherche français ou étrangers, des laboratoires publics ou privés. 


\title{
Evapotranspiration en zone semi-aride de deux couverts végétaux (gazon, blé) obtenue par plu- sieurs méthodes. I. - Evaluation de l'ETP (conditions hydriques non limitantes)
}

\author{
Charles RIOU \& Roger CHARTIER* \\ I.N.R.A., Station de Bioclimatologie-Télédétection, route de St-Cyr, F 78000 Versailles \\ * Mission ORSTOM, 18, avenue Charles-Nicolle, Tunis, Tunisie
}

RÉSUME

Différentes méthodes sont utilisées pour évaluer l'évapotranspiration potentielle du gazon sous le climat semiaride de la Tunisie (région de Tunis) :

1. La mesure directe de l'ETP par un évapotranspiromètre.

2. Les bacs d'évaporation « classe A » et « colorado " en différents sites (gazon irrigué et sol nu).

3. La formule de Penman avec une fonction du vent déterminée à partir des formulations de Riou-ITIER et BRUTSAERT.

Toutes ces méthodes fournissent des résultats comparables satisfaisants.

Une fonction du vent a été élaborée pour le cas du blé et des valeurs d'ETP du blé calculées par la formule de PEnman ; ces valeurs seront comparées aux mesures dans la $2^{\mathrm{e}}$ partie de cet article (VACHAUd et al.).

Mots clés additionnels : Tunisie, bacs d'eau libre, formule de PENMAN, bilan hydrique.

Use of different methods to estimate evapotranspiration from two plant covers (grass and wheat) in the semi-arid zone. I. - Estimation of potential evapotranspiration when soil water is not a limiting factor.

\begin{abstract}
Several methods were used to estimate PET under a semi-arid Mediterranean climate in Tunisia (near Tunis) : 1. measurement of PET by means of a lysimeter : 2. use of colorado and class A pans and correlation with PET ; 3. use of PENMAN's formula with a modified version of Dalton's formula elaborated from RIOU-ITIER and BRUTSAERT relationships. The different methods were compared and found to lead to similar results. Finally, a modified DALTON formula is proposed for calculating wheat-field evaporation; these values will be compared with measurements of water consumption obtained by use of neutron moisture probes and tensiometers in the next paper (VACHAUD et al.).
\end{abstract}

Additional key words : Tunisie, water pan evaporation, PENMAN's formula, water balance.

\section{INTRODUCTION}

Les mesures ont été effectuées sur un site aménagé en 1979, par l'ORSTOM près de Tunis, dans le cadre d'une convention avec le Ministère de l'Agriculture de Tunisie et d'une collaboration avec la Direction des ressources en eau et en sol. Ce site installé dans la plaine du Mornag (RIOU \& CHARTIER, 1982) comprend 3 parcelles : une parcelle de 0,5 ha maintenue sans végétation, un gazon irrigué en période sèche d'une surface équivalente, 1 ha mis en culture (ici en blé). Le climat est méditerranéen et semi-aride (tabl. 2) ; comme on peut le constater, les années hydrologiques (1 $1^{\text {er }}$ septembre-31 août) 1980-1981 et 1981-1982 ont des pluviosités assez proches (451 et $419 \mathrm{~mm})$ et voisines de la moyenne $(450 \mathrm{~mm})$, mais dont la répartition mensuelle est différente et plus favorable à la production agricole au printemps 1982 .

Sur ce site sont effectuées, outre les mesures météorologiques classiques sous abri, celles du rayonnement 
TABLEAU

Liste des symboles.

List of symbols.

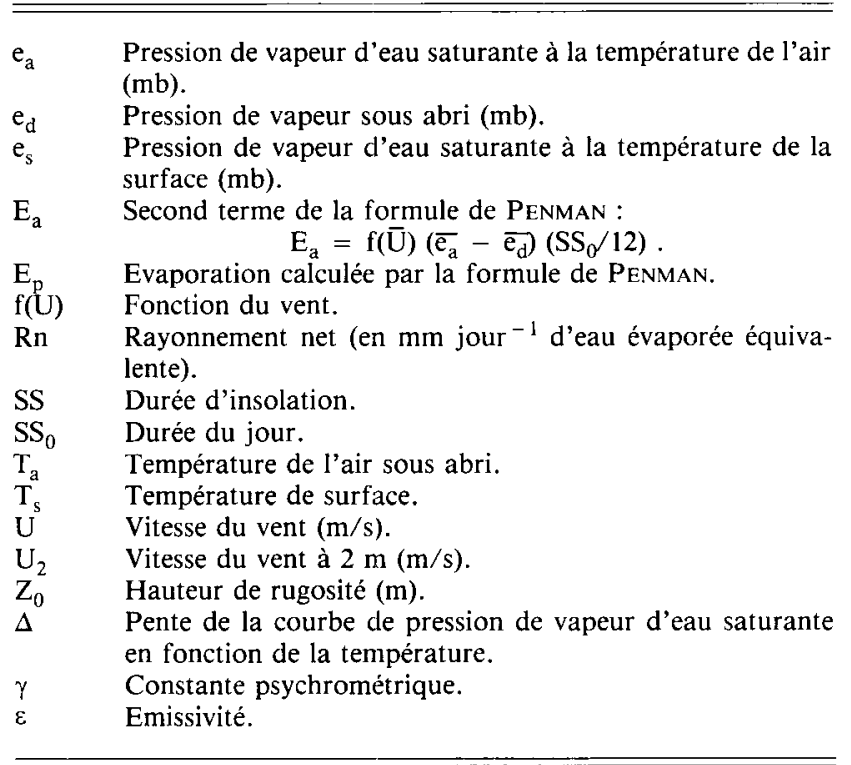

global (pyranomètre thermo-électrique étalonné par un pyrhéliomètre de LINKE-FEUSSNER), du vent, de l'évapotranspiration potentielle du gazon (kikuyu) d'un évapotranspiromètre THORNTHWAITE de $4 \mathrm{~m}^{2}$, de l'évaporation de l'eau libre en bacs (classe A et colorado modifié ORSTOM).

La $1^{\text {re }}$ partie de cet article se propose de comparer les résultats de différentes méthodes d'estimation de l'évapotranspiration quand les conditions hydriques du sol sont considérées comme non limitantes.

La $1^{\text {re }}$ méthode consiste à se référer à l'évapotranspiromètre planté en gazon ; une $2^{\mathrm{e}}$ méthode utilise l'évaporation corrigée des différents bacs utilisés ; en $3^{\mathrm{e}}$ lieu enfin, l'évaporation a été calculée par la formule de Penman (1948) adaptée successivement au gazon et au couvert de blé.

La mesure de l'humidité du sol est également effectuée sur ces parcelles à partir de batteries de tensiomètres et d'humidimètres neutroniques; la description de ce dispositif sera détaillée dans la $2^{\mathrm{e}}$ partie de cet article (VACHAUD et al., 1985).

\section{II. ÉVAPOTRANSPIRATION POTENTIELLE DU GAZON. ÉVAPORATION DES BACS D'EAU LIBRE}

L'évapotranspiromètre de $4 \mathrm{~m}^{2}$ et de $0,8 \mathrm{~m}$ de profondeur est placé au milieu de la parcelle irriguée de
0,5 ha. Une nappe d'eau est maintenue à $0,5 \mathrm{~m}$ de la surface et un arrosage quotidien excédentaire est assuré en dehors des pluies importantes. Le gazon est le kikuyu, très répandu localement, maintenu à une hauteur peu variable par des tontes successives. L'eau drainée est recueillie en totalité, même pendant les épisodes très pluvieux. Deux pluviomètres, placés à la même hauteur que le rebord de l'évapotranspiromètre $(0,10 \mathrm{~m}$ au-dessus du sol) et de part et d'autre de ce dernier, fournissent la hauteur de pluie. Il a été vérifié en zone équatoriale que la moyenne de ces 2 pluviomètres donnait une bonne indication de la pluie interceptée par l'évapotranspiromètre (RIOU, 1975). L'anneau de garde est suffisamment grand pour minimiser de manière satisfaisante l'effet d'advection en été : quand on mentionnera l'ETP, il s'agira donc en fait de la consommation d'eau du gazon bien irrigué telle qu'elle est évaluée à partir de cet appareil.

Les bacs d'eau libre sont de 2 types : le bac « colorado », légèrement modifié, appelé aussi bac « ORSTOM ", qui a $1 \mathrm{~m}^{2}$ de surface, $0,60 \mathrm{~m}$ de profondeur en émergeant du sol de $0,1 \mathrm{~m}$, et le bac « classe $A$ » installé suivant les normes, au-dessus du sol.

Un bac « colorado » et un bac « classe A » sont installés près de l'évapotranspiromètre au milieu de la pelouse, un bac colorado est également placé au milieu de la parcelle de sol nu.

Il faut noter que les mesures de l'évaporation des bacs d'eau libre sont moins précises en période pluvieuse qu'en saison sèche : les pluies accompagnées de vents forts rendent les mesures douteuses. Les journées correspondantes ont donc été éliminées. Dans les autres cas et à la suite d'une étude faite à Brazzaville (RIOU, 1975), des corrections de -3 p. 100 et - 5 p. 100 sont apportées à la pluie mesurée au sol respectivement pour les bacs " colorado pelouse » et " classe A ». Pour le bac « sol nu ", la pluie prise en compte est celle mesurée au sol par le pluviomètre international "Snowdon" placé au centre de son écran maillé.

La comparaison de l'évapotranspiration potentielle du gazon (ETP) avec l'évaporation des bacs a porté sur les moyennes décadaires calculées pendant un peu plus de 2 ans. L'évaporation du bac « classe $\mathrm{A}$ » est notée $\mathrm{E}_{\mathrm{clA}}$, celle du bac « colorado » pelouse $\mathrm{E}_{\mathrm{Cpl}}$, et celle du bac « colorado » sol nu $\mathrm{E}_{\mathrm{Csn}}$; elles sont exprimées en $\mathrm{mm} \cdot \mathrm{j}^{-1}$.

Les équations de régression sont :

- $\mathrm{ETP}=0,60 \mathrm{E}_{\mathrm{clA}}-0,12 ; 75$ couples $; \mathrm{r}=0,98$,

- $\mathrm{ETP}=0,81 \mathrm{E}_{\mathrm{Cpl}}-0,16 ; 77$ couples $; \mathrm{r}=0,99$,

- $\mathrm{ETP}=0,57 \mathrm{E}_{\mathrm{Csn}}-0,03 ; 73$ couples $; r=0,98$,

TABLEAU 2

Répartition mensuelle de la pluie à Tunis-Mornag en 1980, 1981 et 1982.

Monthly rain at Tunis-Mornag during 1980, 1981 and 1982.

\begin{tabular}{|c|c|c|c|c|c|c|c|c|c|c|c|c|c|}
\hline & $\mathrm{J}$ & $\mathrm{F}$ & $\mathbf{M}$ & A & $\mathbf{M}$ & $\mathbf{J}$ & $\mathrm{J}$ & A & $\mathrm{S}$ & $\mathrm{O}$ & $\mathbf{N}$ & $\mathrm{D}$ & $\begin{array}{c}\text { Total } \\
(\mathrm{mm} / \mathrm{an})\end{array}$ \\
\hline 1980 & 58 & 42 & 54 & 40 & 46 & 5 & 0 & 16 & 4 & 30 & 33 & 199 & 527 \\
\hline 1981 & 63 & 42 & 22 & 29 & 22 & 7 & 0 & 0 & 11 & 23 & 16 & 39 & 274 \\
\hline 1982 & 162 & 41 & 54 & 46 & 27 & 0 & 0 & 0 & 36 & 170 & 165 & 71 & 772 \\
\hline
\end{tabular}


équations qui peuvent être simplifiées, en forçant les droites à passer par l'origine, sous la forme :

$$
\begin{aligned}
& \mathrm{ETP}=0,58 \mathrm{E}_{\mathrm{clA}} \text { (fig. 1), } \\
& \mathrm{ETP}=0,78 \mathrm{E}_{\mathrm{Cpl}} \text { (fig. 2), } \\
& \mathrm{ETP}=0,57 \mathrm{E}_{\mathrm{Csn}} \text { (fig. 3). }
\end{aligned}
$$

Le rapport $E_{\text {clA }} / E_{C p l}$ est ici supérieur à 1,30, valeur nettement plus élevée que le rapport constaté en Afrique centrale : 1,11 à 1,15 (RIOU, 1975) ; ceci peut être attribué à la vitesse du vent relativement élevèe dans la région de Tunis (moyennes mensuelles de la vitesse du vent à $2 \mathrm{~m}$ allant de 2 à $4 \mathrm{~m} . \mathrm{s}^{-1}$ contre 1 à $2,5 \mathrm{~m} . \mathrm{s}^{-1}$ en Afrique centrale).

Le bac " colorado sol nu » fournit une évaporation journalière qui peut être supérieure de $50 \mathrm{p} .100$ à celle du bac «pelouse ». Une telle différence est due en

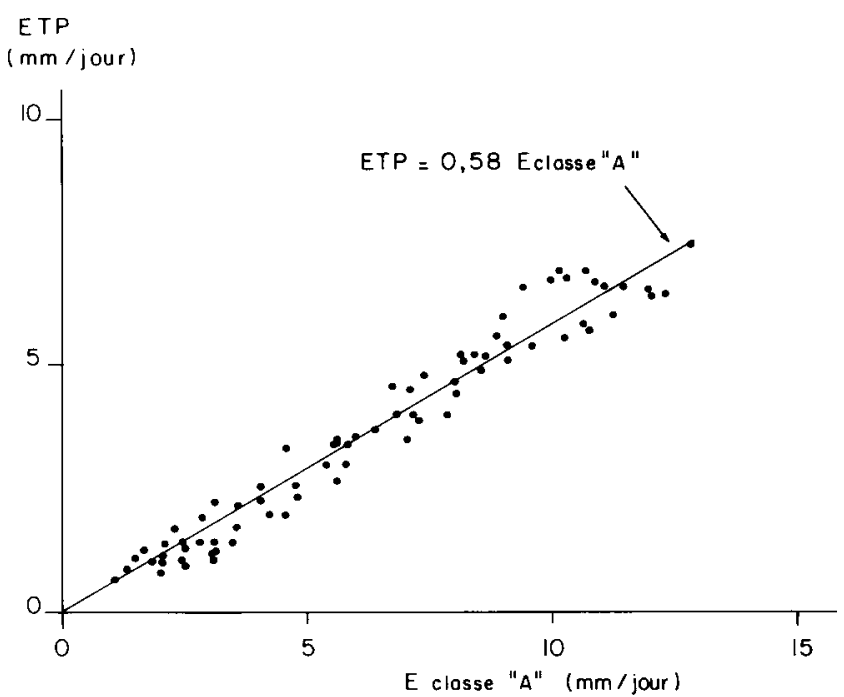

Figure 1

Comparaison des moyennes décadaires d'évaporation du bac « classe $A$ » et de l'ETP.

Ten-day means of pan « class $A$ » evaporation and PET.

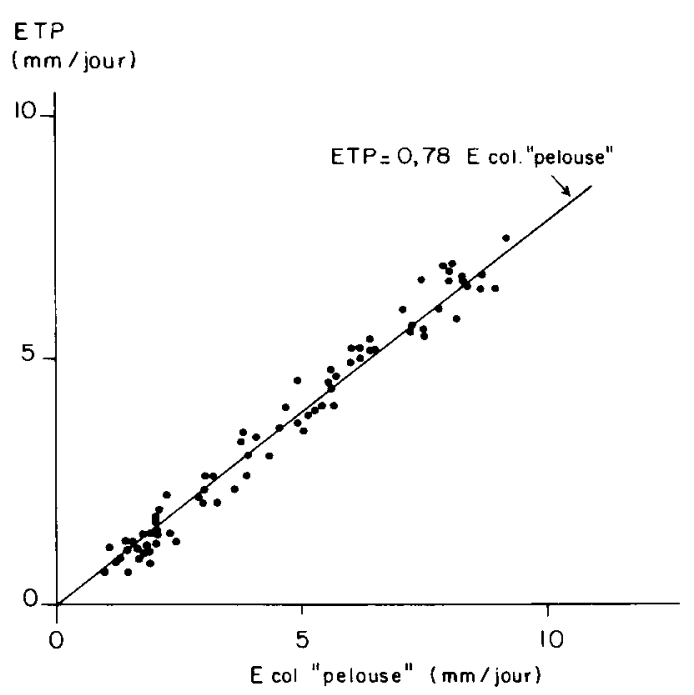

Figure 2

Comparaison des moyennes décadaires d'évaporation du bac « colorado pelouse » et de l'ETP.

Ten-day means of colorado pan (surrounded by lawn) evaporation and PET. grande partie à l'apport de chaleur convective dû au sol nu et sec en surface entourant le bac. A cet effet de bord s'ajoute l'augmentation de vitesse du vent sur une surface de faible rugosité ; la comparaison d'anémomètres placés à $0,25 \mathrm{~m}$ du sol près des bacs montre que la vitesse du vent sur sol nu est fréquemment 1,5 fois plus grande que sur pelouse.

La liaison entre l'ETP et l'évaporation des bacs est ici bien meilleure que celle qui avait été mise en évidence dans le sud de la France (SEGUIN, 1975) ; on ne trouve pas, en particulier, de valeurs d'ETP supérieures à celles du bac colorado.

\section{Cas des mois d'été}

Les équations de régression ont été également établies séparément pour les mois chauds et secs (mai à septembre) :

- $\mathrm{ETP}=0,50 \mathrm{E}_{\mathrm{clA}}+0,84 ; \mathrm{r}=0,86$,

- $\mathrm{ETP}=0,72 \mathrm{E}_{\mathrm{Cpl}}+0,53 ; \mathrm{r}=0,91$,

- $\mathrm{ETP}=0,51 \mathrm{E}_{\mathrm{Csn}}+0,59 ; \mathrm{r}=0,85$.

Les rapports moyens $\mathrm{ETP} / \mathrm{E}_{\mathrm{clA}}, \mathrm{ETP} / \mathrm{E}_{\mathrm{Cpl}}$ et $\mathrm{ETP} / \mathrm{E}_{\mathrm{Csn}}$ sont respectivement $0,57-0,79-0,58$, soit pratiquement identiques aux rapports établis sur l'ensemble de l'année.

Les coefficients de corrélation, un peu moins bons ici que sur l'ensemble de l'année, sont cependant très supérieurs à ceux que SEGUIN (1975) a trouvés à Avignon ; la relation entre l'évaporation du bac colorado pelouse et l'ETP y est également très différente :

$$
\text { Avignon } r=0.72 \quad \mathrm{ETP}=1,82 \mathrm{E}_{\mathrm{Cpl}}-2,36 \text {. }
$$

\section{UTILISATION DE LA FORMULE DE PENMAN}

La formule de PENMAN, $\mathrm{E}_{\mathrm{p}}=\left(\Delta \mathrm{Rn}+\gamma \mathrm{E}_{\mathrm{a}}\right) /(\Delta+\gamma)$, a été modifiée à la suite de plusieurs résultats expérimentaux établis en Tunisie et qui portent sur l'estimation du rayonnement net $\mathrm{Rn}$ et le calcul de la fonction $\mathrm{E}_{\mathrm{a}}$.

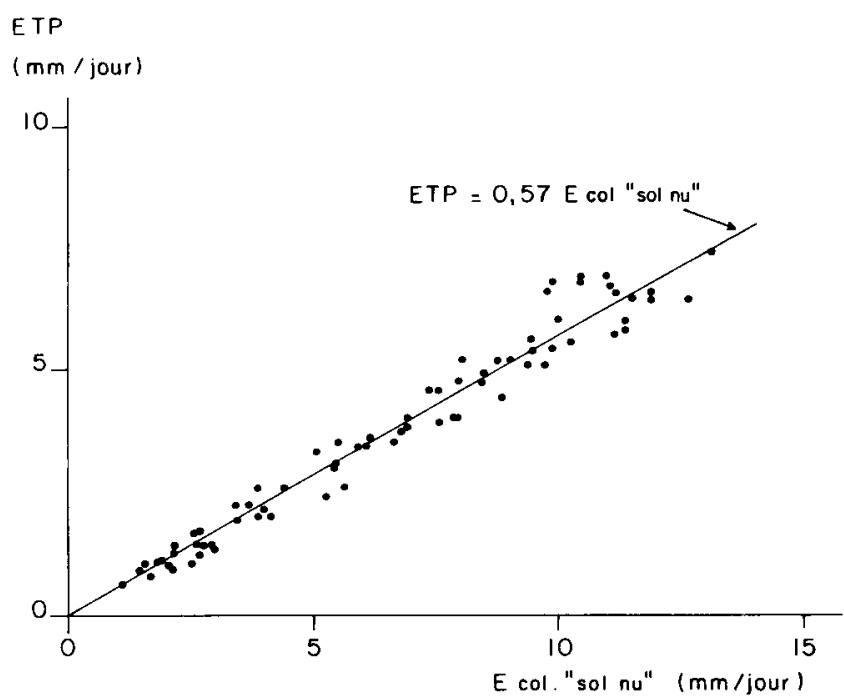

Figure 3

Comparaison des moyennes décadaires d'évaporation du bac « colorado sol nu " et de l'ETP.

Ten-day means of colorado pan (surrounded by bare soil) evaporation and PET. 


\section{A. Calcul du rayonnement net}

Une erreur systématique est commise en saison frâ̂che en Tunisie sur le rayonnement atmosphérique qui est sous-estimé, ce qui entraîne une sous-estimation du rayonnement net (RIOU, 1981).

Par ailleurs, la correction introduite par PENMAN pour tenir compte de la nébulosité est de la forme $0,1+0,9 \mathrm{SS} / \mathrm{SS}_{0}$, alors qu'ici la relation $0,35+0,65 \mathrm{SS} / \mathrm{SS}_{0}$ conviendrait mieux (RIOU, 1981).

En fait, ces 2 erreurs se compensent en hiver, alors qu'en été la correction de nébulosité intervient peu ; il est donc souhaitable de conserver ici la forme habituelle $0,1+0,9 \mathrm{SS} / \mathrm{SS}_{0}$, malgré son imprécision.

Une correction peut en revanche être introduite pour tenir compte de l'écart entre la température de surface $\mathrm{T}_{\mathrm{s}}$ et la température de l'air $\mathrm{T}_{\mathrm{a}}$, sous la forme du terme $\varepsilon\left(\sigma \mathrm{T}_{\mathrm{s}}{ }^{4}-\sigma \mathrm{T}_{\mathrm{a}}{ }^{4}\right) \simeq 4 \varepsilon \sigma \mathrm{T}_{\mathrm{a}}{ }^{3}\left(\mathrm{~T}_{\mathrm{S}}-\mathrm{T}_{\mathrm{a}}\right)$ à ajouter $\mathrm{au}$ rayonnement net ; combinée à l'estimation de la chaleur convective $\gamma \mathrm{f}(\mathrm{U})\left(\mathrm{T}_{\mathrm{s}}-\mathrm{T}_{\mathrm{a}}\right)$, cette correction conduit à la formule finale :

$$
\mathrm{E}_{\mathrm{p}}=\frac{\Delta \mathrm{Rn}+\left[\gamma+4 \varepsilon \sigma \overline{\mathrm{T}}_{\mathrm{a}}^{3} / \mathrm{f}(\mathrm{U})\right]}{\Delta+\gamma+4 \varepsilon \sigma \overline{\mathrm{T}}_{\mathrm{a}}^{3} / \mathrm{f}(\mathrm{U})} \mathrm{E}_{\mathrm{a}}
$$

$E_{p}, R n$ et $E_{a}$ étant exprimés ici en $\mathrm{mm} /$ jour.

\section{B. Calcul de la fonction $f(U)$ dans $E_{a}$}

\section{Cas des bacs}

En ce qui concerne les bacs, la température de l'eau relevée chaque jour à $6 \mathrm{~h} 00$ et 12 h 00 TU permet le calcul des coefficients de la formule de DALTON.

Pour le bac colorado " pelouse », le meilleur ajustement statistique, effectué à partir des valeurs moyennes journalières des paramètres, est :

$$
\begin{gathered}
\mathrm{E}_{\mathrm{Cpl}}=\left(0,10 \overline{\mathrm{U}}_{2}+0,15\right)\left(\overline{\mathrm{e}}_{\mathrm{s}}-\overline{\mathrm{e}}_{\mathrm{d}}\right)\left(\mathrm{SS}_{0} / 12\right) \\
\mathrm{r}=0,80
\end{gathered}
$$

E est en $m m . j^{-1}, U_{2}$ en $\mathrm{m} / \mathrm{s}$, e $e_{\mathrm{s}}$ et $e_{\mathrm{d}}$ en millibars.

C'est cette fonction qui est utilisée ici dans le calcul de la formule de PENMAN ; on note qu'elle s'écarte assez peu des formules traditionnellement proposées pour des petits plans d'eau ou des végétations rases, tout en introduisant le rapport $\mathrm{SS}_{0} / 12$.

$$
\text { PENMAN } E=\left(0,14 \bar{U}_{2}+0,13\right)\left(\overline{\mathrm{e}}_{\mathrm{s}}-\overline{\mathrm{e}}_{\mathrm{d}}\right)
$$

Service hydrologique d'URSS

$$
E=\left(0,10 \overline{\mathrm{U}}_{2}+0,14\right)\left(\overline{\mathrm{e}}_{\mathrm{s}}-\overline{\mathrm{e}}_{\mathrm{d}}\right)
$$

\section{Cas du gazon et du blé}

Dans un article récent, RIOU \& ITIER (1983) proposent, en utilisant des résultats de BRUTSAERT (1975), une méthode de calcul de l'évapotranspiration valable pour un couvert de rugosité dynamique donnée $\mathrm{Z}_{0}$. La formulation peut être simplifiée pour permettre l'évaluation de $\mathrm{E}$ à partir des moyennes journalières $\overline{\mathrm{e}}_{\mathrm{s}}, \overline{\mathrm{e}}_{\mathrm{d}}$, $\overline{\mathrm{U}}_{2}, \overline{\mathrm{T}}_{\mathrm{s}}$ et $\overline{\mathrm{T}}_{\mathrm{a}}$, ce qui conduit à une équation de la forme :

$$
E=\left(a \bar{U}_{2}+b \frac{\bar{T}_{s}-\bar{T}_{a}}{\bar{U}_{2}}\right)\left(S S_{0} / 12\right)\left(\bar{e}_{s}-\bar{e}_{d}\right)
$$

où $\mathrm{E}$ est l'évapotranspiration moyenne journalière et a et $\mathrm{b}$ sont des constantes dépendant de la hauteur de rugosité $Z_{0}$ (une telle expression est valable pour une vitesse de vent moyenne journalière supérieure à $1 \mathrm{~m} / \mathrm{s}$ ).

Les coefficients a et b ont été calculés pour un gazon court $\left(Z_{0}=4 \cdot 10^{-3} \mathrm{~m}\right)$ et un blé bien développé $\left(Z_{0}=3 \cdot 10^{-2} \mathrm{~m}\right)$, ce qui donne pour

$$
\begin{aligned}
& \mathrm{f}\left(\mathrm{U}_{2}\right)=\mathrm{E} /\left[\left(\overline{\mathrm{e}}_{\mathrm{s}}-\overline{\mathrm{e}}_{\mathrm{d}}\right)\left(\mathrm{SS}_{\mathrm{O}} / 12\right)\right]: \\
& \text { gazon } \mathrm{f}\left(\mathrm{U}_{2}\right)=\left(0,09 \mathrm{U}_{2}+0,085 \frac{\overline{\mathrm{T}}_{\mathrm{s}}-\overline{\mathrm{T}}_{\mathrm{a}}}{\overline{\mathrm{U}}_{2}}\right) \\
& \text { blé développé } \mathrm{f}\left(\mathrm{U}_{2}\right)=\left(0,21 \mathrm{U}_{2}+0,155 \frac{\overline{\mathrm{T}}_{\mathrm{s}}-\overline{\mathrm{T}}_{\mathrm{a}}}{\overline{\mathrm{U}}_{2}}\right)
\end{aligned}
$$

Différentes mesures effectuées au-dessus du gazon et du blé ont permis d'évaluer les écarts $\bar{T}_{s}-\bar{T}_{a}$ et de proposer des valeurs moyennes respectives de $3,5^{\circ}$ et $2^{\circ}$, ce qui donne les relations finales :

$$
\text { gazon : } \quad \mathrm{f}\left(\mathrm{U}_{2}\right)=\left(0,09 \overline{\mathrm{U}}_{2}+\frac{0,30}{\overline{\mathrm{U}}_{2}}\right)
$$

blé à l'épiaison :

$$
f\left(U_{2}\right)=\left(0,21 \bar{U}_{2}+\frac{0,31}{\bar{U}_{2}}\right)
$$

La première de ces estimations de $\mathrm{f}(\mathrm{U})$ est voisine de celle qui est proposée par PENMAN ; elle est également proche de la relation établie expérimentalement pour le bac «colorado» (sauf pour les faibles vitesses du vent).

La fonction du vent adoptée pour un blé à son maximum de hauteur fournit par contre des valeurs nettement plus élevées que la précédente $(0,73$ au lieu de 0,37 pour un vent de $3 \mathrm{~m} / \mathrm{s}$ ). En cours de croissance, la fonction $\mathrm{f}(\mathrm{U})$ augmente donc pour le blé ; dans les calculs qui suivent nous nous sommes limités aux 2 cas extrêmes : blé jeune assimilé au gazon, blé en fin de croissance ; les évaluations de la formule de PENMAN dans ces 2 cas doivent donc encadrer les valeurs de l'évapotranspiration maximale du blé.

\section{Résultats}

\section{Evaporation calculée du bac colorado "pelouse 》}

La formule de PENMAN ainsi corrigée et avec un terme $E_{a}$ calculé selon (1) a été d'abord appliquée au calcul de l'évaporation décadaire du bac colorado au sein de la pelouse; les résultats sont indiqués sur la figure 4 qui regroupe plus de 2 années de mesure et sont tout à fait satisfaisants, ce qui constitue ici un bon test pour l'estimation de $\mathrm{Rn}$.

\section{Calcul de l'ETP du gazon}

La même formule, mais en utilisant (2) comme expression de $f(U)$ dans $E_{a}$, permet le calcul de l'ETP du gazon indiquée dans le tableau 3 ; la comparaison avec les valeurs mesurées décadaires est également illustrée par la figure 5 .

La régression portant sur les moyennes mensuelles (ETP en $\mathrm{mm} /$ jour) conduit à la relation :

$\mathrm{ETP}=0,964 \mathrm{ETP}$ calculée $+0,087 ; \mathrm{r}=0,988$. 


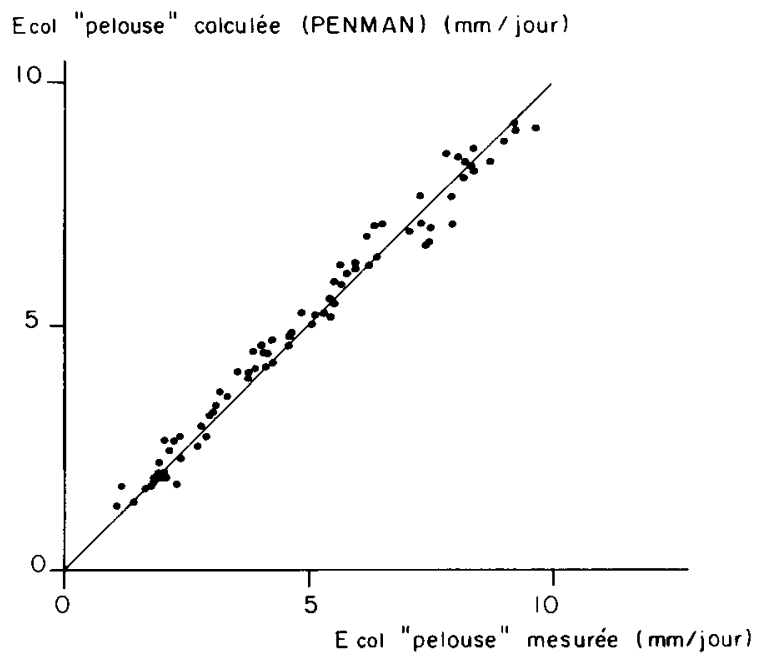

Figure 4

Comparaison des moyennes décadaires d'évaporation du bac « colorado pelouse " calculées (Penman) et mesurées.

Ten-day means of colorado pan (surrounded by lawn) evaporation and calculated data (Penman formula).

Cette relation est meilleure que celle que SEguin (1975) obtint à Avignon, particulièrement en été ; la prise en compte ici de la correction $\varepsilon\left(\sigma \mathrm{T}_{\mathrm{s}}{ }^{4}-\sigma \mathrm{T}_{\mathrm{a}}{ }^{4}\right)$ majore la valeur calculée de l'ETP quand $\mathrm{E}_{\mathrm{a}}$ est supérieur à $\mathrm{Rn}$, soit en période d'advection, et peut expliquer en partie ce meilleur accord observé.

\section{Calcul de l'ETP du blé}

Le tableau 4 compare les moyennes décadaires de l'évapotranspiration obtenues par le calcul pour le
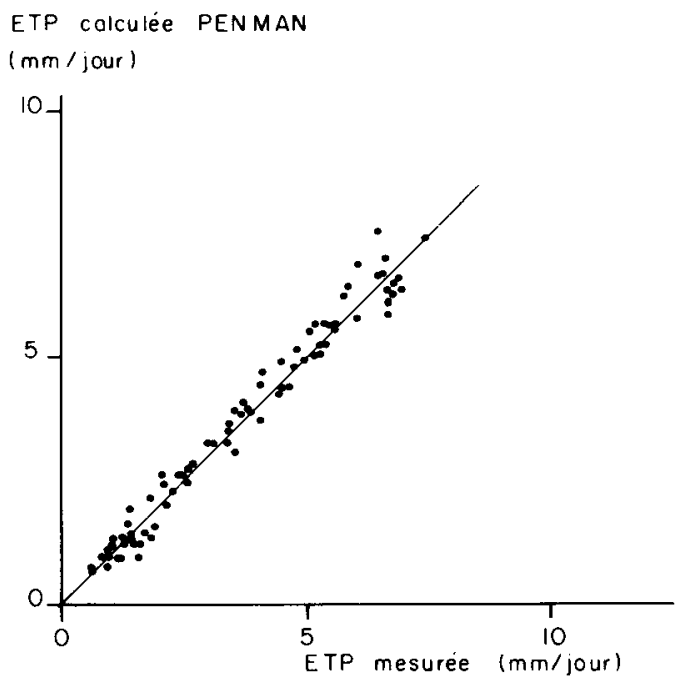

Figure 5

Comparaison des moyennes décadaires de l'évapotranspiration potentielle calculées (Penman) et mesurées.

Ten-day means of PET and calculated data (Penman formula).

gazon $\left(\mathrm{ETP}_{\mathrm{c}}\right.$ gazon) et pour le blé $\left(\mathrm{ETP}_{\mathrm{c}}\right.$ blé), pendant la période de croissance du blé.

En fait, comme il a été indiqué au $\S$ B.2., seules les valeurs calculées de mars et avril peuvent être rapprochées de la consommation maximale du blé ; dans la période qui précède, l'évapotranspiration maximale du blé est intermédiaire entre les valeurs calculées du gazon et du blé.

La comparaison des valeurs du tableau 4 met également en évidence l'importance du terme $\mathrm{E}_{\mathrm{a}}$ dans le

TABLEAU 3

Comparaison des valeurs mensuelles de l'ETP (en mm/mois) calculées (c, PENMAN) et mesurées (m) à Tunis-Mornag (1980 à 1982). Comparison of monthly PET data calculated (c, PENMAN) and measured (m) at Tunis-Mornag (1980-1982).

\begin{tabular}{|c|c|c|c|c|c|c|c|c|c|c|c|c|c|c|}
\hline & & $\mathrm{J}$ & $\mathrm{F}$ & $\mathbf{M}$ & A & M & $\mathbf{J}$ & $\mathbf{J}$ & A & $\mathrm{S}$ & $\mathrm{O}$ & $\mathrm{N}$ & D & Année \\
\hline 1980 & $\begin{array}{l}\mathrm{c} \\
\mathrm{m}\end{array}$ & & & & & & $\begin{array}{l}179 \\
189\end{array}$ & $\begin{array}{l}196 \\
209\end{array}$ & $\begin{array}{l}174 \\
182\end{array}$ & $\begin{array}{l}120 \\
107^{*}\end{array}$ & $\begin{array}{l}80 \\
72^{*}\end{array}$ & $\begin{array}{l}48 \\
44\end{array}$ & $\begin{array}{l}33 \\
31\end{array}$ & \\
\hline 1981 & $\begin{array}{l}\mathrm{c} \\
\mathrm{m}\end{array}$ & $\begin{array}{l}40 \\
31\end{array}$ & 39 & $\begin{array}{l}75 \\
75\end{array}$ & $\begin{array}{l}104 \\
101\end{array}$ & $\begin{array}{l}148 \\
137\end{array}$ & $\begin{array}{l}165 \\
162\end{array}$ & $\begin{array}{l}200 \\
186\end{array}$ & $\begin{array}{l}172 \\
171\end{array}$ & $\begin{array}{l}128 \\
125\end{array}$ & $\begin{array}{l}89 \\
97\end{array}$ & $\begin{array}{l}41 \\
50\end{array}$ & $\begin{array}{l}37 \\
51\end{array}$ & $\begin{array}{l}(1199) \\
(1186)\end{array}$ \\
\hline 1982 & $\begin{array}{l}\mathrm{c} \\
\mathrm{m}\end{array}$ & $\begin{array}{l}38 \\
35\end{array}$ & $\begin{array}{l}38 \\
32\end{array}$ & $\begin{array}{l}68 \\
53\end{array}$ & $\begin{array}{l}76 \\
66\end{array}$ & $\begin{array}{l}126 \\
114\end{array}$ & $\begin{array}{l}198 \\
184\end{array}$ & $\begin{array}{l}209 \\
203\end{array}$ & $\begin{array}{l}173 \\
163\end{array}$ & $\begin{array}{l}120 \\
115\end{array}$ & $\begin{array}{l}71 \\
71\end{array}$ & $\begin{array}{l}39 \\
79\end{array}$ & $\begin{array}{l}24 \\
27\end{array}$ & $\begin{array}{l}1180 \\
1122\end{array}$ \\
\hline
\end{tabular}

* Valeurs par défaut : reprise de la végétation après une coupe rase.

( ) Total annuel - février.

TABLEAU 4

Valeurs décadaires calculées de l'ETP du gazon et de l'ETP du blé, en $\mathrm{mm} / \mathrm{jour}$.

Ten-day values of calculated PET for grass and wheat-field ( $\mathrm{mm} /$ day)

\begin{tabular}{|c|c|c|c|c|c|c|c|c|c|c|c|c|c|c|c|}
\hline \multirow{3}{*}{$\begin{array}{l}\frac{1981}{\text { ETP }_{\mathrm{c}} \text { gazon }} \\
\text { ETP }_{\mathrm{c}} \text { blé }\end{array}$} & \multicolumn{3}{|c|}{ Janvier } & \multicolumn{3}{|c|}{ Février } & \multicolumn{3}{|c|}{ Mars } & \multicolumn{3}{|c|}{ Avril } & \multicolumn{3}{|c|}{ Mai } \\
\hline & 1,30 & 1,45 & 1,15 & 1,25 & 1,30 & 1,65 & 1,95 & 2,40 & 2,85 & 3,25 & 3,20 & 3,95 & 3,85 & 4,70 & 5,65 \\
\hline & 1,90 & 2,00 & 1,45 & 1,80 & 1,75 & 2,25 & 2,45 & 3,30 & 3,65 & 4,20 & 3,95 & 5,00 & 5,05 & 6,10 & 7,65 \\
\hline \multicolumn{16}{|l|}{1982} \\
\hline $\mathrm{ETP}_{\mathrm{c}}$ gazon & 1,35 & 1,40 & 0,95 & 1,20 & 1,60 & 1,25 & 2,00 & 2,45 & 2,10 & 2,70 & 2,25 & 2,65 & 3,25 & 3,95 & 4,90 \\
\hline $\operatorname{ETP}_{\mathrm{c}}$ blé & 2,00 & 2,15 & 1,40 & 1,65 & 2,15 & 1,70 & 2,85 & 3,30 & 2,80 & 3,35 & 2,65 & 3,15 & 3,90 & 4,55 & 5,65 \\
\hline
\end{tabular}


résultat fourni par la formule de PENMAN, la majoration de la fonction $f\left(\bar{U}_{2}\right)$ entraînant une nette augmentation de l'ETP calculée ; dans la $2^{\mathrm{e}}$ partie de l'article, on verra que les mesures neutroniques confirment le dépassement de l'ETP gazon par l'évapotranspiration du blé.

\section{CONCLUSION}

Les résultats font apparaître l'intérêt des mesures en bacs d'évaporation (bac colorado enterré ou bac classe A) pour estimer l'évapotranspiration potentielle d'un gazon court ; plus simple et moins coûteuse, l'installation d'un bac enterré au sein d'une petite parcelle irriguée nous paraît donc tout à fait à conseiller sur un périmètre irrigué.
Par ailleurs, la formule de PENMAN, calculée selon le mode indiqué et avec les fonctions $f(U)$ introduites ici (et justifiées expérimentalement dans le cas de l'évaporation en bac et par voie théorique pour la végétation), rend bien compte des valeurs mesurées de l'évaporation de l'eau libre et de l'ETP du gazon ; en ce qui concerne l'évapotranspiration du blé, seule l'évolution de la réserve en eau du sol, à partir de profils hydriques obtenus à l'humidimètre neutronique, nous permettra de tester la précision du calcul quand les conditions hydriques du sol seront favorables. C'est ce qui sera fait dans la $2^{\mathrm{e}}$ partie de cet article (VACHAUD et al., 1985).

Reçu le 7 février 1984. Accepté le 2 novembre 1984.

\section{RÉFÉRENCES BIBLIOGRAPHIQUES}

Brutsaert W., 1975. The roughness length for water vapor, sensible heat and other scales. J. Atmos. Sci., 32 (10), 2028-2031.

Penman H. L., 1948. Natural evaporation from open water, bare soil and grass. Proc. R. Soc., A, 193, 120-145.

Riou C., 1975. La détermination pratique de l'évaporation. Application à l'Afrique Centrale. Mémoire ORSTOM, 80, 236 p.

Riou C., 1981. Note sur la détermination du rayonnement atmosphérique en Tunisie et ses conséquences sur le calcul de l'évaporation. La Météorologie, VI ${ }^{\mathrm{e}}$ série, 27, 15-18.

Riou C., Chartier R., 1982. La station hydroclimatologique du Mornag. $N^{\circ} l-$ Description et résultats 1980-1981. Evaporation en bac d'eau libre et évapotranspiration potentielle. D.R.E.S. Tunis, ORSTOM Mission en Tunisie, Janvier 1982, 40 p.

Riou C., Itier B., 1983. Expression des coefficients de transfert de la vapeur d'eau du sol à l'atmosphère. La Météorologie, VI ${ }^{\mathrm{e}}$ série, 32 (sous presse).

Seguin B., 1975. Etude comparée des méthodes d'estimation d'ETP en climat méditerranéen du Sud de la France (Région d'Avignon). Ann. agron., 26 (6), 671-691.

Vachaud G., Vauclin M.. Riou C.. Chaabouni Z., 1985. Evapotranspiration en zone semi-aride de deux couverts végétaux (gazon, blé) obtenue par plusieurs miéthodes. II. - Méthodes neutroniques et tensiométriques. Agronomie, 5 (3), 267-274. 